\title{
Respuestas celulares de macrófagos a nanopartículas de óxidos metálicos ${ }^{\diamond}$
}

\section{Macrophage cellular responses to metal oxide nanoparticles}

\author{
Melissa Isabel Gutiérrez-Araujo, ${ }^{*}$ Rafael Vázquez-Duhalt, ${ }^{* *}$ Karla Oyuki Juárez-Moreno**,
}

\begin{abstract}
Recently, metal oxide nanoparticles received special attention due to their broad spectrum of unique properties with applications in research and industry. Because of to the raising of the nanotechnology market, the synthesis and use of nanomaterials are expected to increase considerably in the coming years. Thus, it is crucial to test the effects of nanomaterial exposure in biological systems. To achieve this, nanotoxicology aims to evaluate nanomaterials' toxicity that correlates to the physicochemical parameters such as size and shape. In this review, we focus on macrophages' cellular responses exerted by their exposure to metal oxide nanoparticles. Since macrophages are the first cells to interact with nanoparticles once they enter the body, they are able to uptake them by different mechanisms. The interaction with some metal oxide nanoparticles causes an alteration in the expression of genes that modulate macrophages' phenotype and functions. This modulation can be used for immunotherapy against cancer; however, it is of utmost importance to study whether this modulation leads to immunotoxicity, which directly impacts the primary functions of macrophages, and therefore the immune system.
\end{abstract}

KEYWORDS: nanotoxicology, macrophages, toxicity, oxide metallic nanoparticles.

RESUMEN: Recientemente las nanopartículas de óxidos metálicos han recibido especial atención por su amplio espectro de propiedades únicas que pueden tener múltiples aplicaciones en la investigación y la industria. Debido al incremento del mercado de la nanotecnología, se espera que en los próximos años la síntesis y el uso de nanomateriales aumente considerablemente, por lo cual es de vital importancia evaluar los efectos de la exposición de nanomateriales en sistemas biológicos. Por ello, la nanotoxicología tiene como objetivo evaluar la toxicidad de los nanomateriales, íntimamente ligada a sus parámetros fisicoquímicos como tamaño y forma, entre otros. En esta revisión, nos enfocamos en las respuestas celulares desencadenadas por macrófagos a causa de la exposición a nanopartículas de óxidos metálicos, pues los macrófagos son las primeras células en interaccionar con las nanopartículas una vez que ingresan al organismo, y tienen la habilidad de fagocitarlas por distintos mecanismos. La interacción con algunas nanopartículas óxido metálicas causan alteración en la expresión de genes que modulan el fenotipo y las funciones de los macrófagos. Esta modulación puede ser aprovecha-

Recibido: 30 de octubre de 2020.

Aceptado: 10 de febrero 2021.

• Los autores agradecen al Departamento de Bionanotecnología de CNyN-UNAM, en particular a la Dra. Katrin Quester, al Proyecto de Cátedras Conacyt No. 53 "Nanotoxicología: evaluación toxicológica de los nanomateriales", al proyecto Conacyt 269071 y al Sistema Nacional de Evaluación Toxicológica de Nanomateriales, SINANOTOX.

* Centro de Investigación Científica y de Educación Superior de Ensenada, Posgrado en Nanociencias.

** Universidad Nacional Autónoma de México, Centro de Nanociencias y Nanotecnología, Departamento de Bionanotecnología, Ensenada, Baja California, México.

-Autora de correspondencia: kjuarez@ens.cnyn.unam.mx 
da para inmunoterapia contra el cáncer; sin embargo, es de suma importancia estudiar si esta modulación dirige a inmunotoxicidad, la cual impacta directamente de manera negativa en las funciones primordiales de los macrófagos y, por lo tanto, del sistema inmunológico.

PALABRAS CLAVE: nanotoxicología, macrófagos, toxicidad, nanopartículas de óxidos metálicos.

\section{Introducción}

En años recientes, la nanotecnología ha causado toda una revolución en el ámbito industrial y científico al haber permitido el estudio y la manipulación de las interacciones y fenómenos a nivel atómico y molecular, dando lugar a una nueva generación de materiales nanométricos con propiedades y aplicaciones únicas (Ramsden, 2016). Se denomina nanomaterial a todo aquel material sintetizado de manera natural, incidental o manufacturado con al menos una dimensión igual o menor a $100 \mathrm{~nm}$, y se clasifican de acuerdo con su origen, dimensionalidad, composición química y toxicidad potencial (Dolez, 2015). Para el caso de los nanomateriales, las características fisicoquímicas como tamaño reducido y la gran área superficial les otorgan propiedades ópticas, eléctricas, mecánicas, químicas, térmicas, magnéticas, entre otras, que difieren del material en bulto (micro o macrométrico) y que son de interés para el área industrial (Roduner, 2006); sin embargo, dichas características también impactan en su interacción con sistemas biológicos y su toxicidad. Debido al amplio espectro de aplicaciones de los nanomateriales en múltiples áreas, se estima que para el año 2022 el valor del mercado de la nanotecnología será de aproximadamente 55 mil millones de dólares (Inshakova y Inshakov, 2017).

Uno de los campos más prometedores derivados de la nanotecnología es la bionanotecnología, cuyo objetivo de estudio son las interacciones de nanomateriales con los sistemas biológicos con la finalidad de desarrollar nuevas estrategias de diagnóstico y terapias contra enfermedades que actualmente no tienen cura o tratamiento exitoso. Algunas de estas estrategias incluyen nanoacarreadores de fármacos, biosensores, agentes antimicrobianos, inmunomoduladores, entre otros (Nagamune, 2017). Actualmente, es posible encontrar una gran variedad de productos que contienen nanopartículas de óxidos metálicos en el mercado, ya sea en productos de uso cotidiano, aditivos alimentarios, e incluso medicamentos (Wiechers y Musee, 2010; Song, B. et al., 2016). En este contexto, es un hecho que los nanomateriales son y serán parte de nuestra vida diaria, y, por lo tanto, la síntesis, aplicación y la exposición a nanomateriales se verá considerablemente incrementada en los próximos años; por ello, es de vital importancia evaluar su seguridad y regular su comercialización y disposición final.

\section{La nanotoxicología: ¿qué tan seguro es un nanomaterial?}

La nanotoxicología nace de la necesidad de evaluar la toxicidad de los nanomateriales, uno de sus retos es el de diseñar y adaptar los métodos de análi- 
sis toxicológicos convencionales al estudio de nanomateriales. La toxicidad de los nanomateriales depende de una gran cantidad de factores como su tamaño, forma y propiedades químicas de la superficie, y pueden inducir toxicidad a través del contacto directo, por ingesta de agua o alimentos contaminados, o por su incorporación a productos de uso diario (Kumar et al., 2018).

Las interacciones de los nanomateriales con los sistemas biológicos que conducen a respuestas biológicas tóxicas constan de cuatro fases principales: 1) introducción de nanomateriales al sistema biológico, pudiéndose producir a través de seis vías: intravenosa, dérmica, subcutánea, inhalación, intraperitoneal y oral, la mayor exposición es mediante la vía inhalatoria seguida por la gastrointestinal; 2) adsorción: tiene lugar cuando el nanomaterial interacciona con componentes biológicos como proteínas y células, ocasionando la formación de una corona proteica que cubre el nanomaterial y le otorga una identidad biológica, o el nanomaterial puede ser opsonizado, es decir, puede ser cubierto por moléculas conocidas como opsoninas que tienen como función facilitar la fagocitosis; 3) biodistribución: consiste en la distribución del nanomaterial a través del torrente sanguíneo a varios órganos del cuerpo en donde pueden ser modificados, metabolizados o acumulados, y, 4) excreción y desecho de los nanomateriales, en donde pueden participar órganos como el riñón, hígado o conducto biliar (figura 1). Si los nanomateriales no son excretados se desconoce su comportamiento a largo plazo (Fischer y Chan, 2007).

Una vez absorbidos por el organismo, los nanomateriales pueden interactuar con las células de manera pasiva y activa a través de la regulación de funciones celulares mediante mecanismos moleculares, en los que sus propiedades fisicoquímicas determinan su biocompatibilidad y seguridad. De tal modo, la respuesta celular se caracteriza por variar considerablemente tanto entre distintas líneas celulares como en el nanomaterial de estudio, aún si estos son de la misma naturaleza (Villanueva-Flores et al., 2020). Esto complica la predicción de la toxicidad de un nanomaterial de acuerdo con sus características en un sistema biológico en específico. Algunas de las respuestas citotóxicas desencadenadas por la exposición a nanomateriales comprenden la generación de especies de oxígeno altamente reactivas (ROS, del inglés reactive oxygen species) pudiendo conducir a estrés oxidativo, perturbación mitocondrial, estrés del retículo endoplásmico, degradación y desnaturalización de proteínas, alteración del ciclo celular, daño al ADN, peroxidación de lípidos, entre otras (Nel et al., 2006). La citotoxicidad desencadenada por nanopartículas consiste en 4 mecanismos fundamentales: 1) adhesión a la superficie de la membrana; 2) penetración dentro de la célula y el núcleo; 3) generación de ROS y toxicidad celular, y, 4) modulación de señalización celular (Dakal et al., 2016).

\section{Macrófagos: función e importancia}

Una de las vías de introducción de nanomateriales más estudiadas es la inhalatoria, donde, de manera dependiente del tamaño, es probable que ocurra el 
Mundo Nano | ARTículos DE REVISIóN | www.mundonano.unam.mx

14(27), 1e-16e, julio-diciembre 2021 | https:// doi.org/10.22201/ceiich.24485691e.2021.27.69661

Melissa Isabel Gutiérrez-Araujo, Rafael Vázquez-Duhalt, Karla Oyuki Juárez-Moreno

Figura 1. Análisis de calidad de los estudios incluidos para la síntesis de resultados.

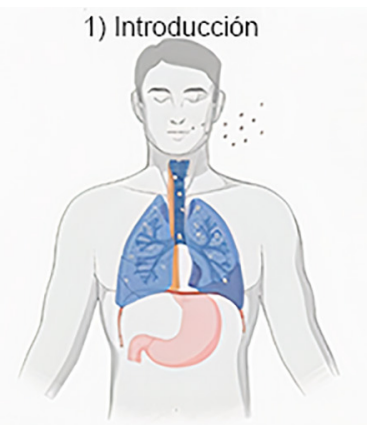

3) Biodistribución
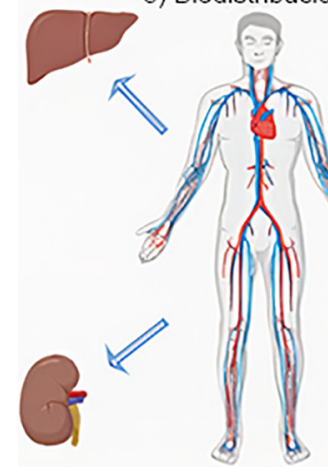

2) Adsorción

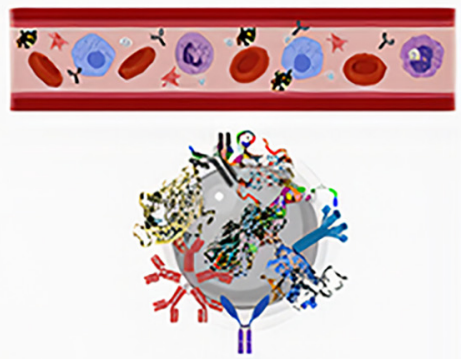

4) Excreción

Nota: El primer paso consiste en la introducción de nanomateriales a través de diversas vías, una de ellas es la inhalatoria (1), en donde son adsorbidos y de esta forma pueden llegar al torrente sanguíneo (2) donde se encuentra una gran variedad de células, proteínas y moléculas. Dadas sus propiedades fisicoquímicas, algunas de estas proteínas y moléculas pueden adsorberse en la superficie, formando una "corona proteica", otorgándole una identidad biológica al nanomaterial que le permitirá internalizarse en algunas células. Mediante esta vía, el nanomaterial puede ser distribuido hacia otros órganos como el hígado, en donde puede ser metabolizado, transformado o bioacumulado (3). Por último, los nanomateriales pueden ser excretados a través de su transporte a los riñones (4). Fuente: Elaboración de los autores (imagen creada en BioRender.com).

depósito de nanomateriales en el sistema respiratorio (INSHT, 2015). Debido a la exposición constante a microrganismos patógenos y agentes exógenos en el sistema respiratorio, en estos tejidos existe una alta concentración de células del sistema inmune innato con capacidades fagocíticas para erradicar y prevenir posibles daños en el hospedero. La función fisiológica del sistema inmunológico consiste en defender al hospedero de microrganismos infecciosos y sustancias foráneas. La respuesta inmunológica contra un microrganismo o agente exógeno se encuentra orquestada primeramente por la inmunidad innata, consistente en una respuesta rápida que carece de especificidad, seguida por la inmunidad adaptativa, que es una respuesta tardía con alta especificidad. La inmunidad innata y adaptativa se encuentran íntimamente ligadas, y en esencia, dependen la una de la otra (Abbas et al., 2015). 
Una de las principales células del sistema inmunológico innato en el sistema pulmonar son los macrófagos y, debido a su capacidad natural de fagocitar nanomateriales, algunos autores sugieren que son los primeros en interaccionar con las nanopartículas, por lo tanto, también en mediar la respuesta inmunológica. Por ello, estudiar el efecto en la función de macrófagos y los mecanismos de reconocimiento de nanopartículas es sumamente importante (Gustafson et al., 2015). Los macrófagos se encuentran distribuidos en una gran variedad de tejidos y desempeñan un papel fundamental en la respuesta inmunológica tanto innata como adaptativa. Algunas de sus funciones incluyen fagocitosis, presentación de antígeno e inducción de la inflamación, así como en el mantenimiento de la homeostasis celular a través de la eliminación de células apoptóticas, reparación de tejidos dañados, entre otras; por lo que, de manera general, los macrófagos, dependiendo de su fenotipo, pueden tener funciones pro-inflamatorias o anti-inflamatorias (Le et al., 2019).

La fagocitosis, además de tener un papel fundamental en la alimentación de la célula, tiene otras funciones como producto de la evolución. Es un proceso activo mediado por receptores dependiente de energía, que permite la internalización en vesículas de partículas de hasta $10 \mu \mathrm{m}$, y comprende las siguientes etapas: 1 ) reconocimiento del microrganismo o agente exógeno mediante receptores de membrana por parte del macrófago, los cuales pueden ser receptores de reconocimiento de patrones, receptores opsónicos o receptores de cuerpos apoptóticos; 2) la membrana de la célula fagocítica sufre una alteración que rodea la partícula a fagocitar; 3) el agente exógeno es ingerido a través de su internalización en una vesícula que recibe el nombre de fagosoma, y, 4) se forma el fagolisosoma a través de la fusión del fagosoma y lisosoma, este último contiene un bajo $\mathrm{pH}$ y enzimas digestivas que producen la destrucción de la partícula (figura 2). Este proceso es crucial para el sistema inmune adaptativo, pues tras la digestión, los macrófagos pueden presentar antígenos a los linfocitos $\mathrm{B}$ y T, y, en consecuencia, responder al estímulo de

Figura 2. Etapas de la fagocitosis de microrganismos o agentes exógenos por macrófagos.
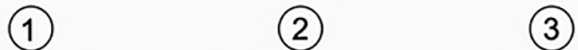

(4)

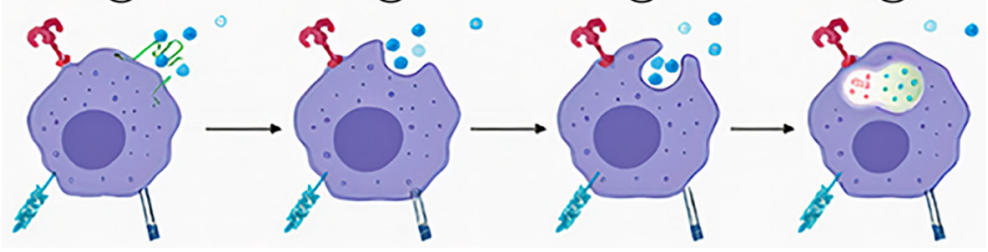

Nota: 1) reconocimiento del agente a fagocitar a través de distintos receptores; 2) la cascada de señalización desencadenada por el reconocimiento dirige a alteraciones en la membrana que permiten rodear al agente a fagocitar; 3 ) el agente es fagocitado dentro de una vesícula que recibe el nombre de fagosoma; 4) el fagosoma se fusiona con el lisosoma, causando la degradación del agente fagocitado mediante enzimas y el bajo $\mathrm{pH}$ del lisosoma.

Fuente: Elaboración de los autores (imagen creada en BioRender.com). 
manera específica y generar memoria contra una posterior invasión por parte del mismo agente (Gordon, 2016). Sin embargo, estos procesos no ocurren posterior a la fagocitosis de nanopartículas y se desconocen los mecanismos moleculares y las consecuencias de la persistencia en los macrófagos debido a la incapacidad de degradarlos a través de enzimas, así como su impacto en las funciones del sistema inmunológico en general.

La fagocitosis es activada a través de cuatro mecanismos moleculares de señalización: fagocitosis mediada por el receptor $\mathrm{Fc}_{\gamma}$, fagocitosis mediada por receptores TLR, fagocitosis mediada por receptores lectina tipo $C$ y fagocitosis mediada por receptores scavenger (Gustafson et al., 2015).

\section{Fagocitosis mediada por el receptor $\mathrm{Fc}_{\gamma}$}

$\mathrm{La}$ activación del receptor $\mathrm{Fc}_{\gamma}\left(\mathrm{Fc}_{\gamma} \mathrm{R}\right)$ es un proceso de replicación a medida que la membrana envuelve la partícula a fagocitar. Esto es posible gracias a la extensión de los pseudópodos que permite encuentros adicionales entre los receptores desocupados y los ligandos disponibles en la superficie de la partícula, lo que al mismo tiempo permite el acercamiento del motivo de activación basado en tirosina inmunorreceptora (ITAM), que es un sustrato para la fosforilación por tirosina cinasas de la familia Src. Seguidamente, se lleva a cabo la incorporación de proteínas adaptadoras que actúan como plataformas para el reclutamiento de componentes de señalización río abajo. Un ejemplo de proteína adaptadora es CrkII, que recluta el complejo entre un factor promotor de la nucleación (Dock180) y un factor intercambiador de nucleótidos de guanina (ELMO1). Los factores promotores de la nucleación activan el complejo de nucleación de actina Arp2/3 a través de Rac1 que, a su vez, provoca la polimerización de actina, proteína del citoesqueleto celular, que impulsa la extensión del pseudópodo, permitiendo la fagocitosis (Flannagan et al., 2012). Los principales factores de transcripción activados son el NFk- $\beta$ y AP-1.

\section{Fagocitosis mediada por receptores TLR}

Los receptores tipo Toll (TLR) son receptores transmembranales de tipo 1. Actualmente se han descrito 10 TLR distintos en humanos con un amplio rango de ligandos que van desde motivos estructurales característicos de microrganismos como bacterias, hongos y parásitos, hasta componentes derivados del propio hospedero. Posterior a la unión del ligando con su receptor TLR, ocurre una dimerización que causa cambios conformacionales necesarios para la señalización río abajo, por la presencia de moléculas adaptadoras cono MyD88, TIRAP/MAL, TRIF, TRAM, cinasas asociadas con el receptor de IL-2 (IRAK), cinasas activadas por el factor transformante beta/TGF- $\beta$ (TAK1), entre otras. La ruta de señalización intracelular promueve la transcripción de genes de citocinas pro-inflamatorias, quimiocinas y coestimuladoras de manera dependiente de la molécula adaptadora río arriba (Mesa-Villanueva y Patiño, 2006). 


\section{Fagocitosis mediada por receptores lectina tipo C}

Los receptores lectina $C$ son un grupo de receptores no opsónicos que reconocen carbohidratos y uno de los más estudiados es el receptor para la manosa MRC1 que reconoce carbohidratos presentes en microrganismos como manosa, fucosa, $\mathrm{N}$-acetilglucosamina y otros ligandos para su eliminación, y sus moléculas adaptadoras incluyen CDC42 y Rho (Gordon, 2016).

\section{Fagocitosis mediada por receptores scavenger}

Entre este grupo de receptores se incluye el receptor scavenger A (SRA-1), receptor de macrófago con estructura de colágeno (MARCO) y CD36. Estos receptores promiscuos se unen a ligandos polianiónicos, tienen una capacidad de señalización pobremente definida, varían en dominio estructural y tienen un reconocimiento distinto, aunque superpuesto, de ligandos apoptóticos y microbianos. En la mayoría de los casos, la participación de CD36 provoca la activación de las tirosinas cinasas de la familia SRC. Después de la unión de lipoproteína oxidada de baja densidad (oxLDL), la activación prolongada de la cinasa de adhesión focal 1 (FAK1), junto con la activación mediada por VAV1 de RAC y la inhibición de la miosina II no muscular, dan como resultado una polimerización de actina, un aumento de la propagación celular y la pérdida de polaridad celular. Otras cascadas de señalización orquestadas por CD36 inducen el reordenamiento de actina y estimulan la producción de citocinas pro-inflamatorias y de señales pro-apoptóticas (Canton et al., 2013).

Estos receptores se diferencian entre sí porque poseen distintos grados de afinidad a un grupo de ligandos, su expresión en los macrófagos varía entre los distintos fenotipos y su activación dirige distintas respuestas inmunológicas las cuales son específicas contra el patógeno a erradicar, lo que impacta de manera directa en su toxicidad. Si bien cualquiera de los procesos moleculares mencionados previamente podría estar involucrado en la internalización de nanopartículas debido a la adsorción de proteínas, se ha observado que los receptores de manosa y $\mathrm{Fc}_{\gamma}$ internalizan nanopartículas de manera más rápida y eficiente que la fagocitosis mediada por receptores scavenger e inclusive se sugiere que más de un grupo de receptores podría causar la internalización en conjunto (Taylor et al., 2005). Sin embargo, debido a la promiscuidad de los receptores scavenger y su capacidad de unir ligandos polianiónicos, este mecanismo en particular resulta de especial interés debido a que permite el reconocimiento por parte del hospedero de materiales extraños como nanopartículas e implantes quirúrgicos, y su internalización por los macrófagos contribuye a la inflamación crónica y a daño progresivo en el tejido. Por ejemplo, un estudio por Arredouani et al. (2005) determinó que partículas de $\mathrm{TiO}_{2}$ fueron reconocidas e internalizadas por macrófagos a través del receptor scavenger MARCO, lo que a su vez ocasionó cambios a nivel de expresión génica en los mismos. Por otro lado, Chao et al. (2012) evidenciaron que la inhibición de receptores scavenger MARCO evita la internalización de nanopartículas de óxido de hierro cubiertas con dextrano. 
Finalmente, las características fisicoquímicas de las nanopartículas determinan el proceso molecular que conlleva su internalización, y, por lo tanto, la respuesta inmunológica orquestada. Entre estas características se encuentran su tamaño, forma y carga superficial (Behzadi et al., 2017). Por lo anterior, diversos autores sugieren que modificar las propiedades fisicoquímicas de las nanopartículas podrían disminuir su internalización en células del sistema inmunológico y con ello, su toxicidad, o bien, dirigir la internalización por una ruta específica con fines terapéuticos. También se han estudiado las estrategias de evasión del sistema inmunológico de algunos microrganismos patógenos, con la finalidad de que puedan ser mimetizados por nanopartículas (Parton y Richards, 2003). Sin embargo, es necesario llevar a cabo más estudios de la relación entre estas características y la internalización, para mejorar nuestro conocimiento y diseñar nanopartículas de manera más específica, para reducir la toxicidad y aumentar la especificidad.

\section{Inmunomodulación de macrófagos por exposición a nanopartículas óxido metálicas}

Con anterioridad se ha planteado el papel y la importancia del sistema inmunológico para el adecuado funcionamiento del organismo; no obstante, una respuesta inmunológica adecuada depende de un delicado equilibro. En condiciones patológicas como en algunas enfermedades autoinmunes, se presenta una exacerbación de la respuesta inmunológica y una falta de tolerancia hacia lo propio que causa daños en el hospedero, por lo cual el tratamiento consiste en tratar de disminuir dicha respuesta, a este proceso se le conoce como inmunosupresión. Por otro lado, el desarrollo de vacunas y los recientes avances en la inmunoterapia contra el cáncer tienen como objetivo estimular la respuesta inmunológica, por ello, se define como inmunoestimulación. De manera general, tanto la inmunosupresión como la inmunoestimulación son tipos de inmunomodulación, que consiste en optimizar la respuesta inmunológica. Las capacidades inmunomoduladoras de algunas nanopartículas óxido metálicas han sido estudiadas para poder aprovecharlas en el área de la inmunología (Kubackova et al., 2019). A continuación, se describen algunas estrategias bionanotecnológicas para el uso de nanopartículas en la inmunoterapia.

\section{Modulación de macrófagos para inmunoterapia en cáncer}

La inmunoterapia consiste en aprovechar y potenciar la habilidad natural del sistema inmunológico para combatir enfermedades de distinta naturaleza. Resultados prometedores se han obtenido en la investigación de la inmunoterapia como tratamiento contra algunos tipos de cáncer, con el objetivo de superar los obstáculos que impone el tumor al evadir y controlar las células inmunitarias. En este sentido, el sistema inmunológico puede inhibir o promover el crecimiento tumoral. Por ello, se propone el uso de inmunomoduladores para potenciar la respuesta inmunológica; asimismo, se requiere modu- 
lar de manera selectiva las células inmunitarias. Debido a la gran plasticidad fenotípica que poseen los macrófagos, estos pueden adoptar distintas funciones en respuesta a las señales del microambiente, lo que se conoce como polarización de macrófagos (Perisé, 2013). Los macrófagos asociados con tumores (TAM) han sido ampliamente estudiados como blancos terapéuticos en la inmunoterapia contra el cáncer, al localizarse en el microambiente natural del tumor, y se ha observado que pueden tener un papel tanto antitumoral como protumoral y que difieren de las funciones de los macrófagos presentes en el tejido sano. En los primeros estadios de la formación del tumor, se reclutan monocitos y macrófagos que se polarizan hacia un fenotipo conocido como M1, caracterizado por tener efectos antitumorales, y conforme el tumor avanza hacia un estadio avanzado, los macrófagos M1 se transforman en M2, que poseen efectos protumorales y participan en la supresión de la respuesta inmunológica (Mantonovani et al., 2017). Algunas nanopartículas óxido metálicas presentan la capacidad de modular estos fenotipos y las actividades de los TAM (figura 3).

Figura 3. Polarización del fenotipo de macrófagos asociados con tumores (TAM) por nanopartículas de óxidos metálicos como estrategia de inmunoterapia.

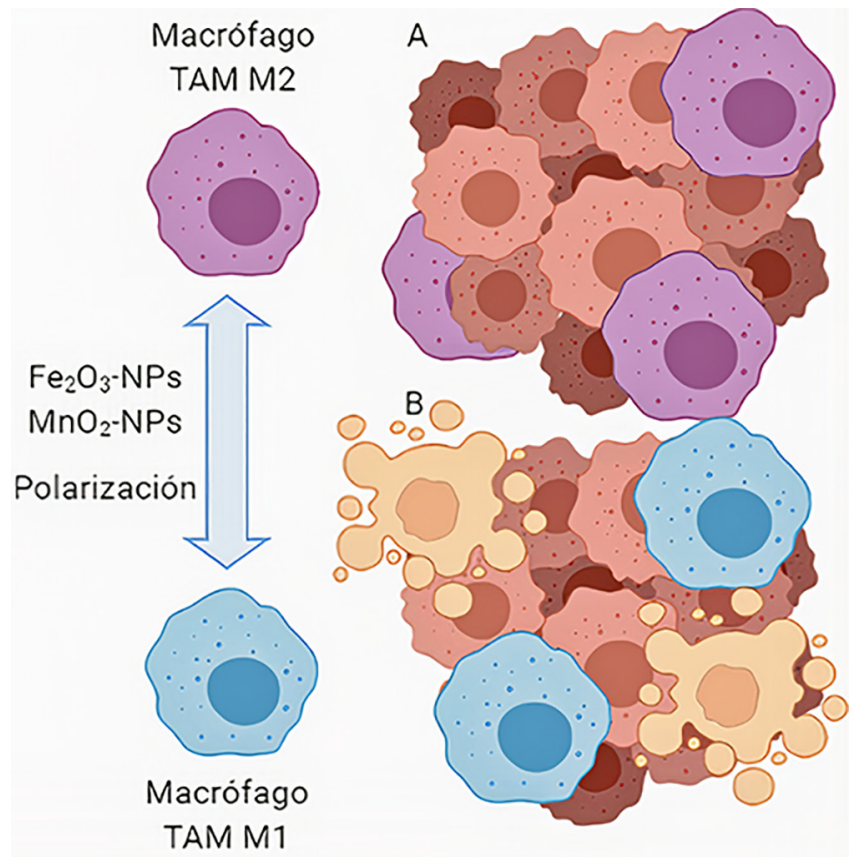

Nota: Nanopartículas de óxido de hierro $\left(\mathrm{Fe}_{2} \mathrm{O}_{3}-\mathrm{NPs}\right)$ y dióxido de manganeso $\left(\mathrm{MnO}_{2}-\mathrm{NPs}\right)$ cubiertas con ácido hialurónico pueden causar cambios a nivel de expresión genética que conducen en un cambio de fenotipo de M2 (anti-inflamatorio) a un fenotipo M1 (pro-inflamatorio) para disminuir la tasa de crecimiento del tumor.

Fuente: Elaboración de los autores (imagen creada en BioRender.com). 
Por ejemplo, en 2016, Zanganeh et al. observaron que el medicamento Ferumoxytol, compuesto por nanopartículas de óxido de hierro aprobadas por la Administración de Medicamentos y Alimentos de los Estados Unidos de América (FDA, por sus siglas en inglés) para el tratamiento de las deficiencias de hierro, tiene un efecto terapéutico intrínseco en tumores, ya que células tumorales coinyectadas con Ferumoxytol exhibieron un considerable retraso en la tasa de crecimiento tumoral en comparación con la inyección de células sin Ferumoxytol. Además, se observó una mayor presencia de macrófagos pro-inflamatorios con fenotipo M1 en el tumor. Estudios posteriores demostraron que el tratamiento con Ferumoxytol causó el aumento de la expresión de genes involucrados en respuestas pro-inflamatorias. Los autores sugieren que este tipo de compuesto modula el fenotipo TAM a través de la reacción Fenton, en donde el peróxido de hidrógeno secretado por los macrófagos M1 podría reaccionar con el hierro para producir radicales hidroxilos tóxicos. En otro estudio, también se reportó que la entrega dirigida de nanopartículas de dióxido de manganeso conjugadas con manano y recubiertas con ácido hialurónico a macrófagos asociados con tumor incrementó la oxigenación tumoral y causó la polarización de macrófagos del fenotipo M2 al M1 (Song, M. et al., 2016).

\section{Respuestas celulares de macrófagos a la exposición a nanopartículas óxido metálicas: ¿modulación o inmunotoxicidad?}

A pesar de los resultados prometedores en la inmunoterapia con el uso de diferentes nanopartículas óxido metálicas, diversos autores han reportado que la exposición de macrófagos a nanopartículas óxido metálicas induce respuestas de inmunotoxicidad, entre las que se encuentran la inducción de la inflamación, internalización de las nanopartículas, perturbación de las funciones fagocíticas, aumento en la producción de ROS y óxido nítrico, entre otras (figura 4) (Nel et al., 2006). La inmunotoxicidad se define como todo efecto adverso sobre la estructura o función del sistema inmune, o en otros sistemas, a consecuencia de la disfunción inmune. Por lo tanto, un efecto adverso o inmunotóxico afecta la inmunidad humoral o celular necesaria para que el hospedero pueda suscitar una respuesta adecuada para su defensa (inmunosupresión) o que cause daño tisular innecesario (autoinmunidad, hipersensibilidad o inflamación crónica) (FDA, 1999). En este contexto, hay que tener en cuenta que, si bien un nanomaterial puede presentar propiedades sumamente interesantes y con potencial terapéutico, es sumamente importante no perder de vista su toxicidad, pues esta última limita su aplicación.

Durante los últimos años, diversos grupos de investigación han enfocado sus esfuerzos en tratar de elucidar los mecanismos toxicológicos de los nanomateriales; sin embargo, los resultados en ocasiones son contradictorios debido en parte a las diferencias en las características fisicoquímicas de los nanomateriales evaluados. Varios autores han reportado la toxicidad in 
vitro en macrófagos expuestos a distintas concentraciones de nanopartículas de óxido de zinc ( $\mathrm{ZnO}-\mathrm{NPs}$ ) de distinto tamaño y concuerdan en que las nanopartículas de menor tamaño y carga positiva presentan mayor toxicidad, la cual es dependiente de la concentración y el tiempo. Además, se ha reportado que existe un incremento en la producción de las citocinas proinflamatorias IL-1b, TNF- $\alpha$ e IL-8, lo cual sugiere activación inmunológica (Feltis et al., 2012). Por otro lado, en 2014, Wang et al. evidenciaron que la solubilidad de los iones $\mathrm{Zn}^{2+}$ es dependiente del $\mathrm{pH}$ del medio, de manera que, a menor $\mathrm{pH}$, mayor concentración de iones $\mathrm{Zn}^{2+}$. Esto sugiere que el potencial tóxico de las $\mathrm{ZnO}-\mathrm{NPs}$ podría verse incrementado en los macrófagos, ya que, como se mencionó anteriormente, el fagolisosoma formado después de la fagocitosis de agentes exógenos posee un bajo $\mathrm{pH}$; por esto se ha especulado que la toxicidad de las $\mathrm{ZnO}$-NPs se debe principalmente a la liberación de iones $\mathrm{Zn}^{2+}$ producto de su disociación.

Por otro lado, se han utilizado las herramientas ómicas para estudiar de manera más general las respuestas celulares suscitadas por la exposición de los macrófagos a las $\mathrm{ZnO}-\mathrm{NPs}$. Ejemplo de ello es un estudio del perfil transcriptómico de macrófagos humanos expuestos a ZnO-NPs de 15 y $12 \mathrm{~nm}$ de diámetro conducido en el año 2013 por Tuomela et al., en donde se estableció que los principales procesos biológicos afectados fueron regulación del crecimiento, muerte celular, desarrollo y control del sistema inmune. Por otro lado, el análisis proteómico reveló alteraciones en rutas implicadas en estrés oxidativo que podrían conducir a genotoxicidad y una fuerte respuesta en rutas de degradación de proteínas (Triboulet et al., 2014). No obstante, elucidar un mecanismo molecular que explique los efectos tóxicos e inflamatorios de las nanopartículas óxido metálicas en macrófagos requiere aún de más investigación.

En este contexto, en 2014, Roy et al. evidenciaron que el aumento de ROS es causado por la disminución y la inhibición de la actividad de enzimas antioxidantes, como consecuencia de la supresión del factor de transcripción Nrf2, dirigiendo a la peroxidación lipídica y de proteínas. Otros estudios llevados a cabo por el mismo grupo de investigación indican que $\mathrm{ZnO}$-NPs de aproximadamente $50 \mathrm{~nm}$ presentan propiedades adyuvantes al alérgeno ovoalbúmina en ratones $\mathrm{Balb} / \mathrm{c}$. Además, describieron que este efecto involucra las vías de señalización mediadas por receptores tipo Toll y Src, debido al incremento en la expresión de TLR2, 4 y 6, así como la proteína de respuesta primaria de diferenciación mieloide 88 (MyD88), la cinasa 1 asociada con el receptor de IL-1 (IRAK-1) y el factor 6 asociado con TNFR (TRAF-6). Todo esto se atribuye a respuestas inflamatorias por el reclutamiento y activación de moléculas de adhesión y células inflamatorias (Roy et al., 2014). Si bien los autores sugieren que este mecanismo podría utilizarse para desarrollar estrategias para su uso terapéutico, es necesario considerar los efectos adversos que podrían desencadenarse por la exposición a las $\mathrm{ZnO}-\mathrm{NPs}$ de $50 \mathrm{~nm}$ en pacientes sanos debido al potencial inmunomodulador de este tipo de NPs. 
Mundo Nano | ARTículos DE REVISIóN | www.mundonano.unam.mx

14(27), 1e-16e, julio-diciembre 2021 | https://doi.org/10.22201/ceiich.24485691e.2021.27.69661

Melissa Isabel Gutiérrez-Araujo, Rafael Vázquez-Duhalt, Karla Oyuki Juárez-Moreno

Figura 4. Respuestas celulares de macrófagos expuestos a nanopartículas de óxido de zinc (ZnO-NPs).

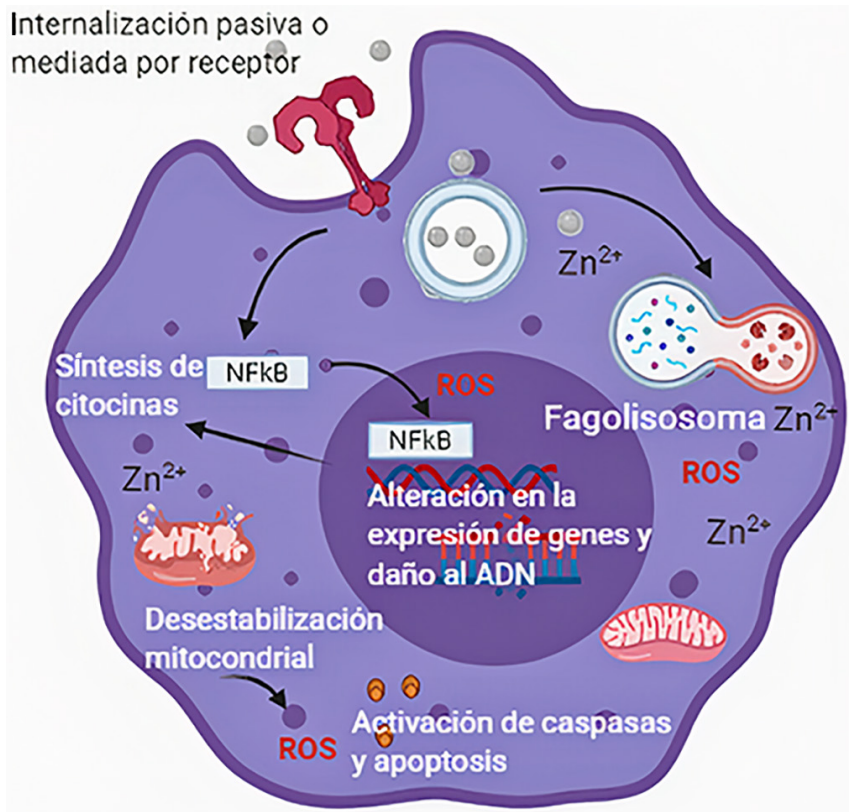

Nota: La internalización de ZnO-NPs puede ocurrir de manera directa por daño a la membrana celular, o bien, puede ser mediada por receptores. La endocitosis de ZnO-NPs forma un fagosoma que se fusiona con un lisosoma, en donde se degrada la cubierta de las nanopartículas y se incrementa la liberación de iones zinc, los cuales se consideran los causantes de su toxicidad. Una vez en el citoplasma, las ZnO-NPs pueden modular de manera directa o indirecta vías de señalización que podrían conducir a la activación de la ruta inflamatoria NF-kB, lo que a su vez ocasionará cambios en la expresión de genes y respuestas celulares. Por otro lado, las ZnO-NPs tienen la capacidad de alterar la función mitocondrial y conducir la activación de caspasas y la inducción de la muerte celular por apoptosis a través de diversos mecanismos como el incremento en la producción de especies reactivas de oxígeno (ROS). Fuente: Elaboración de los autores (imagen creada en BioRender.com).

$\mathrm{Al}$ igual que con las $\mathrm{ZnO}-\mathrm{NPs}$, múltiples estudios sugieren que la rápida disolución y la liberación de iones $\mathrm{Cu}^{2+}$, el tamaño y la forma son los principales factores que influyen en la toxicidad de las nanopartículas de óxido de cobre (CuO-NPs) (Bucchianico et al., 2013). Debido a su evidente potencial tóxico, los autores sugieren que las $\mathrm{CuO}$-NPs podrían ser buenos candidatos como control positivo en ensayos de nanotoxicología. Asimismo, los ensayos in vivo evidencian el potencial tóxico de las CuO-NPs. Por ejemplo, Gosens et al., en 2016, realizaron un estudio en ratas, a las que se les administraron $\mathrm{CuO}-\mathrm{NPs}$ de un tamaño promedio de $14 \mathrm{~nm}$. Después de 5 días de exposición a CuO-NPs, las ratas presentaron inflamación pulmonar, además, los análisis histopatológicos indicaron alveolitis, bronquiolitis, vacuolación del epitelio respiratorio y enfisema pulmonar. Los efectos adversos a causa de la toxicidad fueron desapareciendo en un periodo de 3 semanas post-exposición. 
Por otra parte, se ha discutido sobre el impacto en la reactividad inmunológica de la administración o inhalación de este tipo de nanopartículas. En un estudio realizado en el año 2019 por Holan et al., analizaron la composición de poblaciones celulares de la inmunidad innata y adaptativa en el bazo presentes en ratones expuestos a una inhalación continua de $\mathrm{CuO}-\mathrm{NPs}$ de $30 \mathrm{~nm}$ durante 3 meses. Los resultados demostraron que la inhalación de las $\mathrm{CuO}-$ NPs afecta de forma más severa las células de la inmunidad innata, pues hubo cambios en la proporción de eosinófilos, neutrófilos, macrófagos y células presentadoras de antígeno, mientras que el impacto en las células de inmunidad adaptativa, como linfocitos $\mathrm{T}$ y $\mathrm{B}$, fue mínimo. Esto sugiere que existe un efecto modulador de las $\mathrm{CuO}-\mathrm{NPs}$ dependiente del tiempo de inhalación en la producción de citocinas de las células del sistema inmune adaptativo. Los efectos adversos en las células del sistema inmune por la exposición a $\mathrm{CuO}-\mathrm{NPs}$ pone en evidencia su capacidad de causar inmunotoxicidad.

Lo anterior evidencia el efecto citotóxico, genotóxico e inmunotóxico de las $\mathrm{ZnO}-\mathrm{NPs}$ y las $\mathrm{CuO}-\mathrm{NPs}$ en distintos modelos in vitro e in vivo; sin embargo, es necesario realizar más estudios que establezcan escenarios más reales (concentración y tiempo de exposición), para comprender y lograr elucidar los mecanismos involucrados en dichos efectos adversos; y de este modo, diseñar estrategias que permitan evitar la toxicidad de nanomateriales y aprovechar sus propiedades únicas.

\section{Conclusiones}

Las nanopartículas óxido metálicas presentan una gran variedad de propiedades de interés para la investigación y la industria, por ello se exploran sus usos potenciales. Debido a sus capacidades inmunomoduladoras se ha sugerido su uso para el tratamiento de disfunciones inmunológicas; sin embargo, es necesario considerar el posible efecto tóxico. Los macrófagos son un buen modelo de estudio de inmnunotoxicidad debido a sus funciones primordiales para el mantenimiento del organismo y la orquestación de la respuesta inmunológica. Además, se ha sugerido que son las primeras células en interaccionar con los nanomateriales una vez que estos ingresan al organismo. $\mathrm{Si}$ bien diversos estudios han probado que las nanopartículas óxido metálicas pueden inducir inmunotoxicidad en macrófagos tanto in vitro como in vivo, es necesario realizar más investigación que permita elucidar los mecanismos precisos de toxicidad. Este conocimiento es indispensable para el diseño de nanomateriales mediante la modificación de sus propiedades fisicoquímicas con la finalidad de ampliar su potencial y así pueda ser aprovechado en futuras aplicaciones biomédicas, específicamente en el campo de la inmunomodulación y el combate a enfermedades crónicas como el cáncer. 


\section{Referencias}

Abbas, A., Lichtman A., Pillai S. (2015). Cellular and molecular immunology, 8a ed. España: Elsevier Inc.

Arredouani, M. S., Palecanda A., Koziel H., Huang Y. C., Imrich A., Sulahian T. H., Ning Y. Y., Yang Z., Pikkarainen T., Sankala M., Vargas S. O., Takeya M., Tryggvason K., Kobzik L. (2005). MARCO is the major binding receptor for unopsonized particles and bacteria on human alveolar macrophages. Journal of immunology, 175(9): 6058-64. https://doi.org/10.4049/jimmunol.175.9.6058

Behzadi, S., Serpooshan V., Tao W., Hamaly M. A., Alkawareek M. Y., Dreaden E. C., Brown D., Aiklany A. M., Farokhzad O. C., Mahmoudi M. (2017). Cellular uptake of nanoparticles: journey inside the cell. Chemical Society Reviews, 46(14): 4113-4376. https://doi.org/10.1039/C6CS00636A

Bucchianico, S., Fabbrizi M., Misra S., Valsami-Jones E., Berhanu D., Bergamaschi E., Migliore L. (2013). Multiple cytotoxic and genotoxic effects induced in vitro by differently shaped copper oxide nanomaterials. Mutagenesis, 28(3): 287-299. https://doi.org/10.1093/mutage/get014

Canton, J., Neculai D., Grinstein S. (2013). Scavenger receptors in homeostasis and immunity. Nature Reviews Immunology, 13(9): 621-34. https://doi.org/10.1038/ nri3515

Chao, Y., Makale M., Karmali P. P., Sharikov T., Tsigelny I., Merkulov S., Kesari S., Wrasidio W., Ruoslahti E., Simberg D. (2012). Recognition of dextran-superparamagnetic iron oxide nanoparticle conjugates (Feridex) via macrophage scavenger receptor charged domains. Bioconjugate Chemistry, 23(5): 10031009. https://doi.org/10.1021/bc200685a

Dakal, T. C., Kumar A., Majumdar R. S. y Yadav V. (2016). Mechanistic basis of antimicrobial actions of silver nanoparticles. Frontiers of Microbiology, 7: 18311840. https://doi.org/10.3389/fmicb.2016.01831

Dolez, P. I. (2015). Nanomaterials definitions, classifications, and applications in nanoengineering: Global approaches to health and safety issues. Amsterdam: Elsevier, 3-40.

FDA (Food and Drug Administration). (1999). Immunotoxicity testing guidance. EUA.

Feltis, B., Okeefe S., Harford A., Piva T., Turney T., Wright P. (2012). Independent cytotoxic and inflammatory responses to zinc oxide nanoparticles in human monocytes and macrophages. Nanotoxicology, 6(7): 757-765. https://doi.org/ 10.3109/17435390.2011.620718

Fischer, H. C. y Chan W. C. N. (2007). Nanotoxicity: The growing need for in vivo study. Current Opinion in Biotechnology, 18(6): 565-571. https://doi.org/10.1016/j.copbio.2007.11.008

Flannagan, R., Jaumouillé V., Grinstein S. (2012). The cell biology of phagocytosis. Annual Review of Patholgy; Mechanisms ofDisease, 7: 61-98. https://doi.org/10.1146/ annurev-pathol-011811-132445

Gordon, S. (2016). Phagocytosis: an immunobiologic process. Immunity, 44: 463475. https://doi.org/10.1016/j.immuni.2016.02.026 
Gosens, I., Cassee F., Zanella M., Manodori L., Brunelli A., Costa A., Bokkers B., Jong W., Brown D., Hristozov D., Stone V. (2016.) Organ burden and pulmonary toxicity of nano-sized copper (II) oxide particles after short-term inhalation exposure. Nanotoxicology, 10(8): 1084-1095. https://doi.org/10.3109/174353 90.2016.1172678

Gustafson, H. H., Holt-Casper D., Grainger D. W., Ghandehari H. (2015). Nanoparticle uptake: The phagocyte problem. Nano Today, 10(4): 487-510. https://doi. org/10.1016/j.nantod.2015.06.006

Holan, V., Javorkova E., Vrbova K., Vecera Z., Mikuska P., Coufalik P., Kulich P., Skoupy R., Machala M., Zajicova A., Rossner P. (2019). A murine model of the effects of inhaled $\mathrm{CuO}$ nanoparticles on cells of innate and adaptative immunity - a kinetic study of a continuous three-month exposure. Nanotoxicology, 13(7): 1-12. https://doi.org/10.1080/17435390.2019.1602679

Inshakova, E., Inshakov O. (2017). World market for nanomaterials: structure and trends. International Conference on Modern Trends in Manufacturing Technologies and Equipment. 129, 02013.

INSHT (Instituto Nacional de Seguridad e Higiene en el trabajo). (2015). Seguridady salud en el trabajo con nanomateriales. Madrid, España: Ministerio de Empleo y Seguridad Social.

Kubackova, J., Zbytovska J., Holas O. (2019). Nanomaterials for direct and indirect immunomodulation: A review of applications. European Journal Pharmaceutical Sciences, 15(142): 105139. https://doi.org/10.1016/j.ejps.2019.105139

Kumar, V., Dasgupta N., Ranjan S. (2018). Nanotoxicology: toxicity evaluation, risk assessment and management. CRC Press Taylor y Francis Group.

Le, Q., Yang G., Wu Y., Jang H., Shokouhimehr M., Oh Y. (2019). Nanomaterials for modulating innate immune cells in cancer immunotherapy. Asian Journal of Pharmaceutical Sciences, 14:16-29. https://doi.org/10.1016/j.ajps.2018.07.003

Mantovani, A., Marchesi F., Malesci A., Laghi L., Allavena P. (2017). Tumour-associated macrophages as treatment targets in oncology. Nature Reviews Clinical Oncology, 14(7): 399-416. https://doi.org/10.1038/nrclinonc.2016.217

Mesa-Villanueva, M., Patiño P. J. (2006). Receptores tipo Toll: entre el reconocimiento de lo no propio infeccioso y las señales endógenas de peligro. Inmunología, 25(2): 115-130.

Nagamune, T. (2017). Biomolecular engineering for nanobio/bionanotechnology. Nano convergence, 4(9): 1-56. https://doi.org/10.1186/s40580-017-0103-4

Nel, A., Xia T., Madler L., Li N. (2006). Toxic potential of materials at the nanolevel. Science, 311: 622-627. https://doi.org/10.1126/science.1114397

Parton, R. G., Richards A. A. (2003). Lipid rafts and caveolae as portals for endocytosis: new insights and common mechanism. Traffic, 4: 724-738. https://doi. org/10.1034/j.1600-0854.2003.00128.x

Perisé, B. 2013. Efecto del VIH-1 en la desregulación de los linfocitos B. Papel de dendrímeros carbosilano en la respuesta inflamatoria, como agentes transfectantes y en la polarización de macrófagos de tipo M2. Tesis de doctorado. Universidad Autónoma de Madrid, España, 177. 
Ramsden, J. J. (2016). Nanotechnology an introduction. 2a ed. Cambridge: Elsevier Inc., 358.

Roduner, E. (2006). Size matters: why nanomaterials are different. Chemical Society Reviews, 35: 583e92. https://doi.org/10.1039/b502142c

Roy, R. Kumar D., Sharma A., Gupta P., Chaudhari B., Tripathi A., Das M., Dwivedi P. (2014). ZnO nanoparticles induced adjuvant effect via toll-like receptors and Src signaling in Balb/c mice. Toxicology Letters, 230: 421-433. https://doi. org/10.1016/j.toxlet.2014.08.008

Song, B., Zhang Y., Liu J., Feng X., Zhou T., Shao L. (2016). Is neurotoxicity of metallic nanoparticles the cascades of oxidative stress? Nanoscale Research Letters, 11: 291. https://doi.org/10.1186/s11671-016-1508-4

Song, M., Liu T., Shi C., Zhang X., Chen X. (2016). Bioconjugated manganese dioxide nanoparticles enhance chemotherapy response by priming tumour-associated macrophages toward M1-like phenotype and attenuating tumor hypoxia. ACS Nano, 10(3): 633-647. https://doi.org/10.1021/acsnano.5b06779

Taylor, P. R., Martinez-Pomares L., Stacey M., Lin H. H., Brown G. D., Gordon S. (2005). Macrophage receptors and immune recognition. Annual Review of Immunology, 23:901-44. https://doi.org/10.1146/annurev.immunol.23.021704.115816

Triboulet, S., Aude-García C., Armand L., Gerdil A., Diemer H., Proamer F., CollinFaure V., Habert A., Strub J., Hanau D., Herlin N., Carriére M., Dorsselaer A., Rabilloud T. (2014). Analysis of cellular responses of macrophages to zinc ions and zinc oxide nanoparticles: a combined targeted and proteomic approach. Nanoscale, 6: 6102-6114. https://doi.org/10.1039/C4NR00319E

Tuomela, S., Autio R., Thurnherr T., Arslan O., Kunzmann A., Andersson-Willman B., Wick P., Mathur S., Scheynius A., Krug H., Fadeel B., Lahesmaa R. (2013). Gene expression profiling of immune-competent human cells exposed to engineered zinc oxide or titanium dioxide nanoparticles. PLOS ONE, 8(7): e68415. https://doi.org/10.1371/journal.pone.0068415

Villanueva-Flores, F., Castro-Luego A., Ramirez O., Palomares L. (2020). Understanding celular interactions with nanomaterials: towards a rational of medical nanodevices. Nanotechnology, 31:13200219 pp. https://doi.org/10.1088/13616528/ab5bc8

Wang, B., Zhang Y., Mao Z., Yu D., Gao C. (2014). Toxicity of ZnO nanoparticles to macrophages due to cell uptake and intracellular release of zinc ions. Journal of Nanoscience and Nanotechnology, 14: 5688-5696. https://doi.org/10.1166/ jnn.2014.8876

Wiechers, J., Musee N. (2010). Engineered inorganic nanoparticles and cosmetics: facts, issues, knowledge gaps and challenges. Journal of Biomedical Nanotechnology, 6(5): 408-431. https://doi.org/10.1166/jbn.2010.1143

Zanganeh, S., Hutter G., Spitler R., Lenkov O., Mahmoudi M., Shaw A., Pajarinen J. S., Nejadnik H., Goodman S., Moseley M., Coussens L. M., Daldrup-Link H. E. (2016). Iron oxide nanoparticles inhibit tumour growth by inducing pro-inflammatory macrophage polarization in tumour tissues. Nature Nanotechnology, 11(11): 986-994. https://doi.org/10.1038/nnano.2016.168 